\title{
Analysis And Study On Distribution Of Defect Electric Field On Basin-type Insulator In GIS
}

\author{
Qikun Feng*, and Nianqin Dai \\ Beijing Key Laboratory of High Voltage \& EMC, North China Electric Power University, 102206 Beijing, China
}

\begin{abstract}
With the increase of electric network voltage in power system, the internal discharge phenomenon of gas insulated substation (GIS) during operation often happens. In this paper, a 3-D simulation model of GIS with metal protrusion on basin-type insulator was utilized to study the effect of metal protrusion on the electric field distortion in GIS. It can be concluded from simulation results that the electric field increases with the height of metal protrusion when the radius is constant, while decrease with the increase of radius of metal protrusion. In addition, the metal protrusion near high voltage conductor lead to more severe electric field distortion than that near grounded enclosure. This work is of significance for the actual operation and maintenance of GISs in the power system.
\end{abstract}

\section{Introduction}

Gas insulated substation (GIS) has been rapidly developed in the power system, due to the advantages of high reliability, fast installation and convenient operation $^{[1]}$. However, some metal protrusion will be produced inevitably in the cavity of GIS during the GIS installation and operation. These metal protrusion and suspended particles can cause distortion of the electric field and excessive local electric field inside GIS, which lead to the corona discharge and breakdown ${ }^{[2]}$. Especially, with the increase of electric voltage and capacity in power system, even small defects can lead to internal failures in compact GIS equipment. Insulation defects are increasingly becoming the main cause of equipment failure for GIS. Figure 1 shows the discharge phenomenon caused by the presence of metal protrusion inside the GIS. Therefore, it is important to analyse the electric field distribution when the metal protrusion exist in GIS, which is helpful to reduce the failure rate of GIS and ensure the reliable operation of the power system.

In the research of the distribution of electric field inside the GIS, references $3-5$ is mainly about electromagnetic numerical simulation of basin-type insulators under different shapes when GIS is in stable operation. These calculations are all in an ideal state without the effect of metal protrusion. Moreover, references 6-8 is studying the effect of suspended particles on the electric field distortion in GIS chamber. The suspended particles studied are mainly concentrated near the basin-type insulators. 2-D axisymmetric model was utilized in reference 9 to investigate the distribution of electric field in GIS with the exit of metal protrusion. However, the axisymmetric model is proved to be inappropriate when considering the metal protrusion.
Three-dimensional electric field simulation model is used to calculate the local electric field intensity distortion with the exit of metal protrusion in GIS in this paper. The finite element method is utilized to simulate the electric field when metal protrusion of different sizes and shapes located in different positions on the basintype insulator.

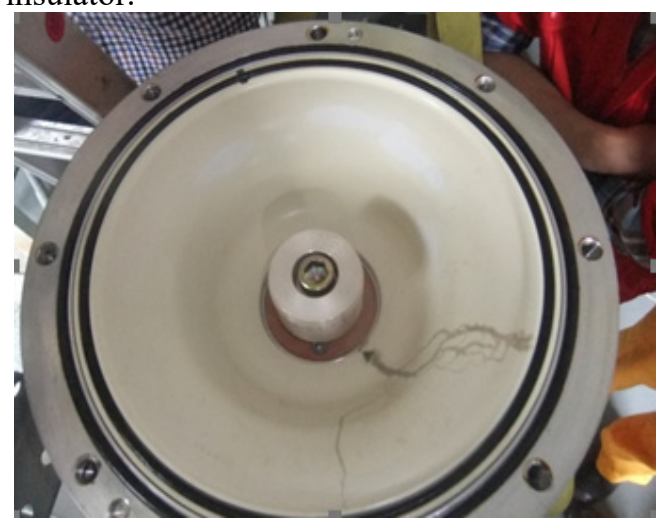

Fig. 1. Discharge traces on basin-type insulator in GIS.

\section{Simulation model}

Figure 2 dedicates 3-D simulation model of basin-type insulator in GIS without the metal protrusion.

\subsection{Governing equations}

A three-dimensional simulation model was established when considering the existence of metal protrusion in GIS. And the governing equations are expressed as follows:

$$
\begin{gathered}
\nabla \cdot D=\rho \\
\nabla \times E=0
\end{gathered}
$$

\footnotetext{
* Corresponding author: fengqikun@ncepu.edu.cn
} 


$$
\begin{gathered}
D=\varepsilon E \\
\nabla^{2} \varphi=-\frac{\rho}{\varepsilon}
\end{gathered}
$$

where $D$ is the displacement vector, $\rho$ is the electric charge density, $E$ is the electric field intensity, $\varepsilon$ is permittivity, $\varphi$ is the electric potential.

Laplace equation is utilized for areas with no electric charge.

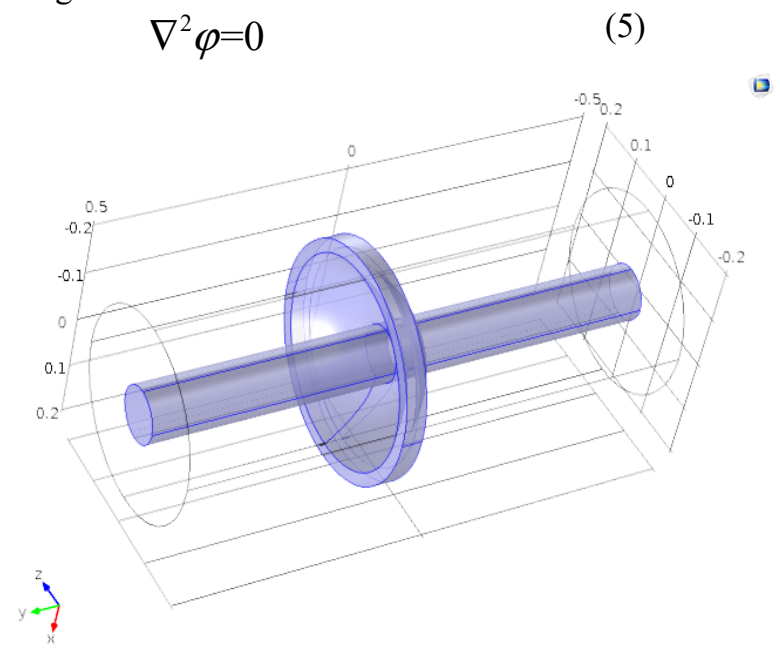

Fig. 2. 3-D model of basin-type insulator in GIS.

\subsection{Meshing grid of the model}

The calculation procession of finite element method mainly includes three parts: pre-processing, model calculation and post-processing. Then the pre-processing includes the establishment of model and meshing. The meshing of simulation model is a key step in determining the accuracy of model calculation.

Table 1. Element dimension parameters of mesh.

\begin{tabular}{|c|c|}
\hline Properties of gird & Values \\
\hline $\begin{array}{c}\text { Maximum unit size } \\
(\mathrm{mm})\end{array}$ & 10 \\
\hline $\begin{array}{c}\text { Minimum size } \\
(\mathrm{mm})\end{array}$ & 0.1 \\
\hline $\begin{array}{c}\text { Maximum unit } \\
\text { growth rate }\end{array}$ & 1.3 \\
\hline $\begin{array}{c}\text { Curvature factor } \\
\text { Resolution of } \\
\text { narrow area }\end{array}$ & 0.2 \\
\hline
\end{tabular}

If the overall mesh is too sparse, the metal protrusion object may not be meshed. However, if the grid is too dense, it will lead to a huge increase in computing time, resulting in unnecessary waste, and even unable to calculate because of the lack of computer memory. In order to solve the above issues, COMSOL software is utilized in this paper. The simulation software uses a combination of virtual boundary surface and multi-layer boundary to mesh the established geometric model. The multi-layer boundary method is a kind of grid local refinement method used in COMSOL software. Applying multi-layer boundary locally in the model can refine the local grid and improve the calculation accuracy. The entire field contains 1625801 units, and the specific settings are shown in table 1 . The partial grid of mesh is shown in figure 3 .

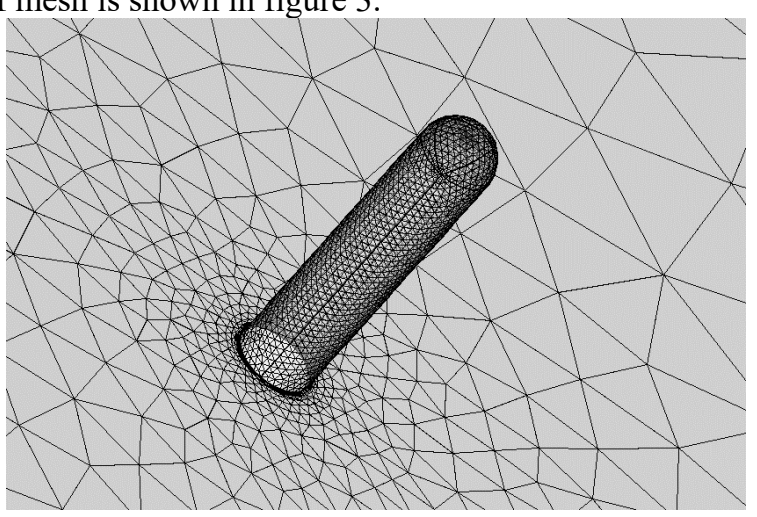

Fig. 3. Local grid of mesh.

\subsection{Calculation procedure}

The simulation calculation in this paper is divided into two parts: the distributions of electric field when the metal protrusion is located on the convex side and concave side of the basin-type insulator.

Firstly, a 3-D model of GIS with the exist of metal protrusion was established. The material of the highvoltage conductor and metal protrusion is made of copper, while the gas in GIS is set to be $\mathrm{SF}_{6}$. And the breakdown field strength of $\mathrm{SF}_{6}$ under different pressures is shown in table 2 . Then, the boundary conditions and loads were applied in the model to get the simulation results. The voltage applied on the high voltage conductor is fixed at $220 \mathrm{kV}$. Next, change the position and size of the metal protrusion, and repeat the previous calculation step to obtain the distribution of electric field intensity generated by different locations and different size metal protrusion.

Table 2. The critical field strength of $\mathrm{SF}_{6}$.

\begin{tabular}{|c|c|}
\hline Pressure (MPa) & $\begin{array}{c}\text { Electric field } \\
\text { strength (V/m) }\end{array}$ \\
\hline 0.2 & $1.77 \times 10^{7}$ \\
\hline 0.4 & $3.54 \times 10^{7}$ \\
\hline 0.6 & $5.31 \times 10^{7}$ \\
\hline
\end{tabular}

\section{Results and discussion}

The simulation results of metal protrusion located at different positions are compared and analysed in the 
following part. The different positions of metal protrusion are shown in figure 4.

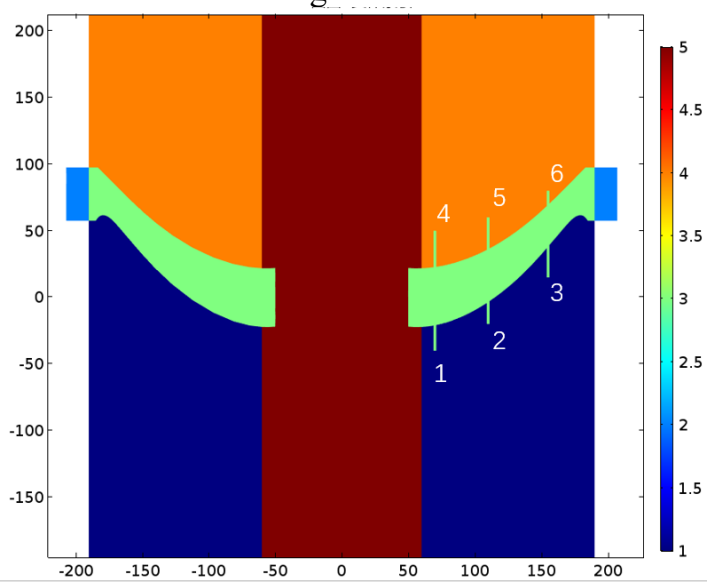

Fig. 4. The position of metal protrusion on basin-type insulator.

\subsection{Analysis of metal protrusion on the convex side}

On the convex side of basin-type insulator, positions 1, 2 and 3 are chosen to study the effect of metal protrusion on the distortion of electric field. In order to avoid the interference, when metal protrusion at one specific location is studied, no other metal protrusion exists at other locations. As shown in figure 5, the electric field intensity increases sharply in the presence of metal protrusion. The value of electric field near metal protrusion is 10 times more than other locations.

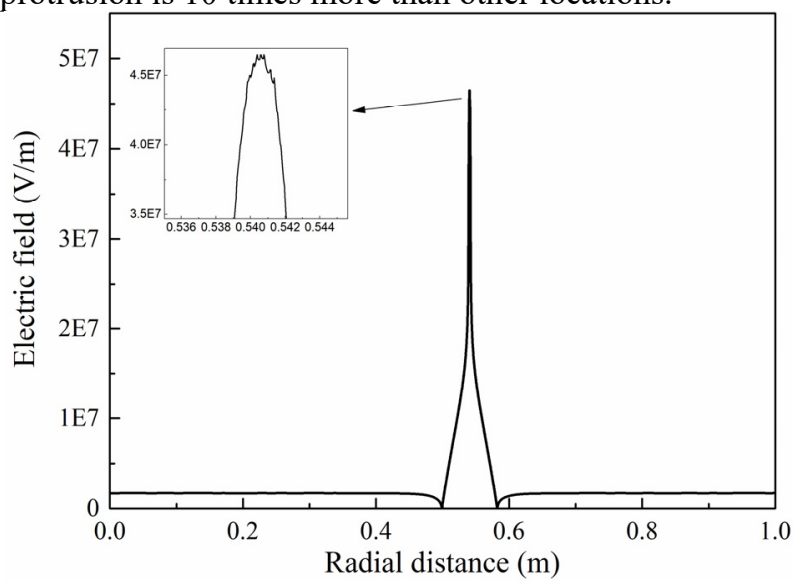

Fig. 5. The electric field distribution around metal protrusion.

Then the position and size of metal protrusion have been changed to study the variation of electric field. As shown in figure 4 , the simulation results of different positions on convex side are investigated. We summarize the distortion of electric field under different metal protrusion, and the corresponding results are obtained as shown in figures 6 and 7.

Through the analysis of calculated results, we found that the electric field increases with the height of metal protrusion when the radius is constant. While the electric field increases with the decrease of radius of metal protrusion when the height is constant. In addition, the intensity of the electric field generated by the same metallic foreign body at different positions also varies greatly.

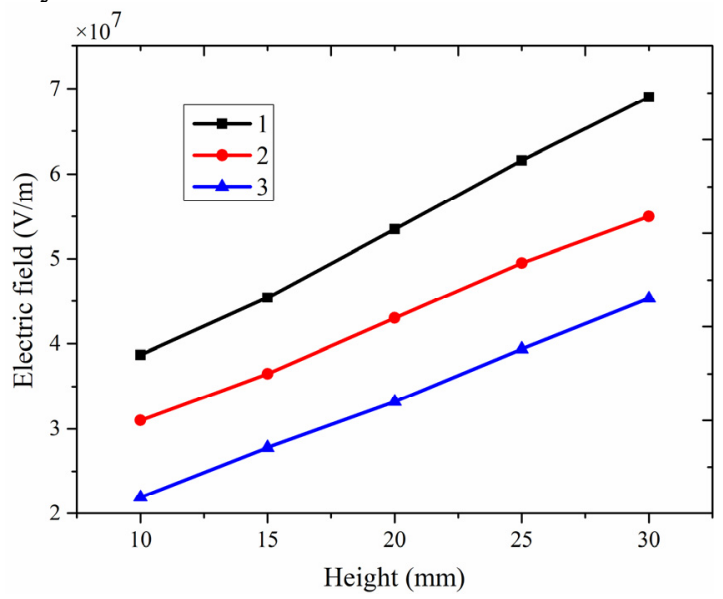

Fig. 6. Influence of metal protrusion height of convex side on

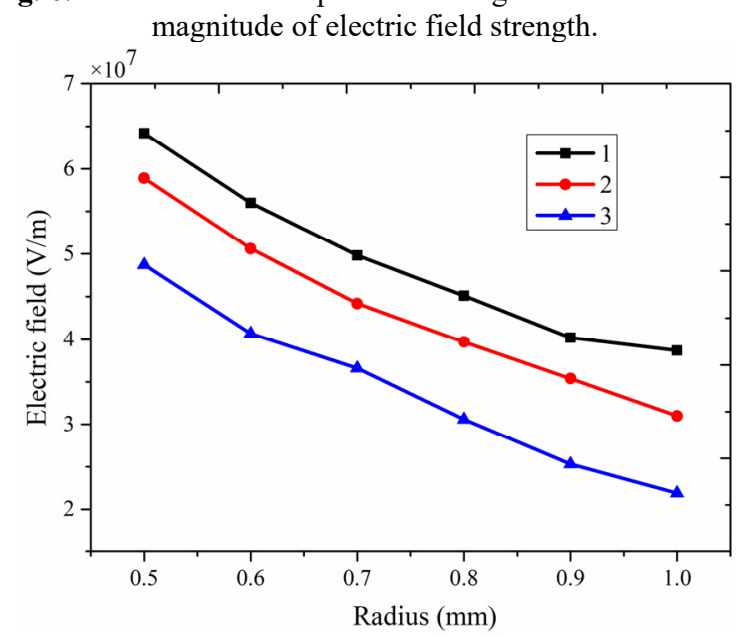

Fig. 7. Influence of metal protrusion radius of convex side on magnitude of electric field strength.

\subsection{Analysis of metal protrusion on the concave side}

As previous study in the above section, the effect of metal protrusion in the concave side on the distortion of electric field is analysed. The effect of height and radius of metal protrusion under different positions are studied in figures 8 and 9. As shown in figure 4 , the position selected on the concave side are marked as position 4,5 and 6.

As for the metal protrusion on the concave side, the local electric field intensity generated also satisfies the basic relationship: the electric field increases with the height of metal protrusion when the radius is constant, while decrease with the increase of radius of metal protrusion when the height is constant. 


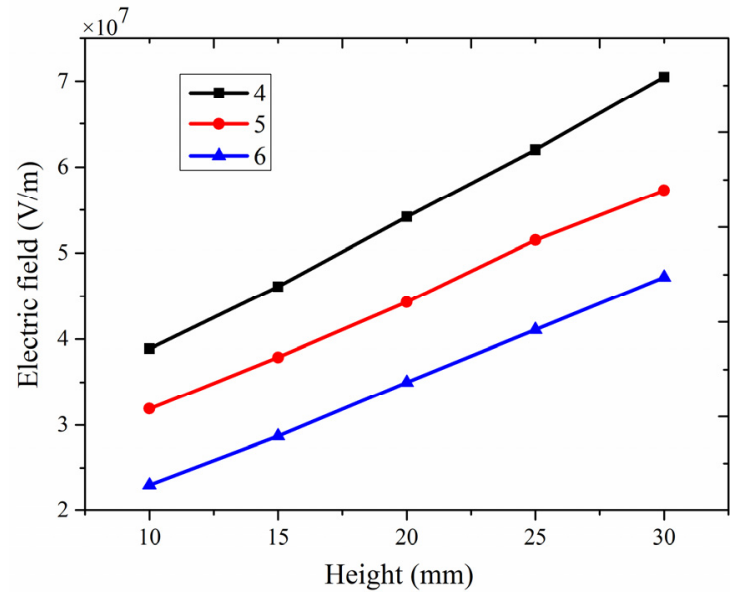

Fig. 8. Influence of metal protrusion height of concave side on magnitude of electric field strength.

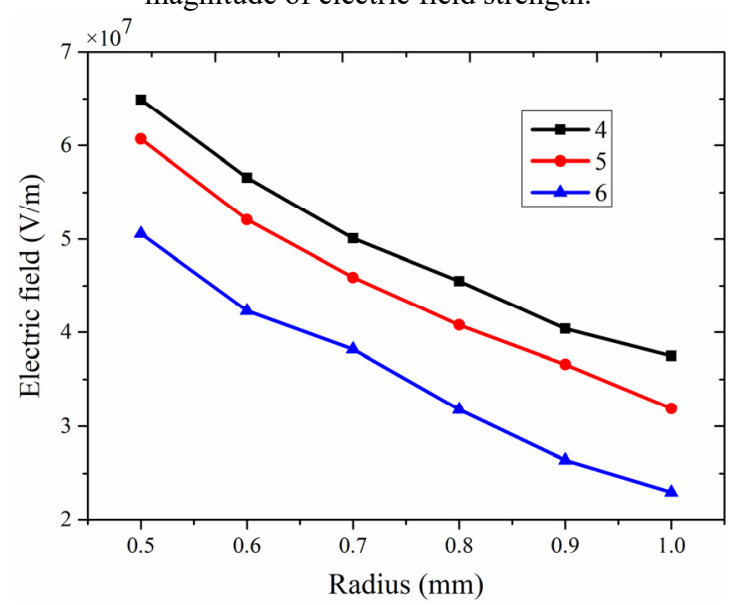

Fig. 9. Influence of metal protrusion radius of concave side on magnitude of electric field strength.

\subsection{Comparative analysis of metal protrusion on two sides}

In order to study the different effect of metal protrusion on convex and concave side of basin-type insulator, the metal protrusion of same size at corresponding position, like position 1 and 4, is discussed in this part.
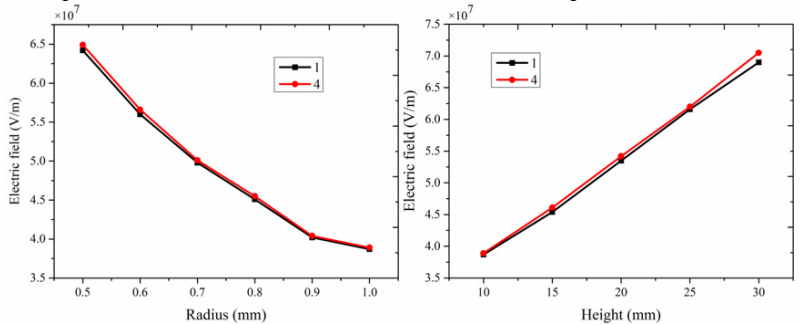

Fig. 10. Comparative study of electric field at position 1 and 4 .
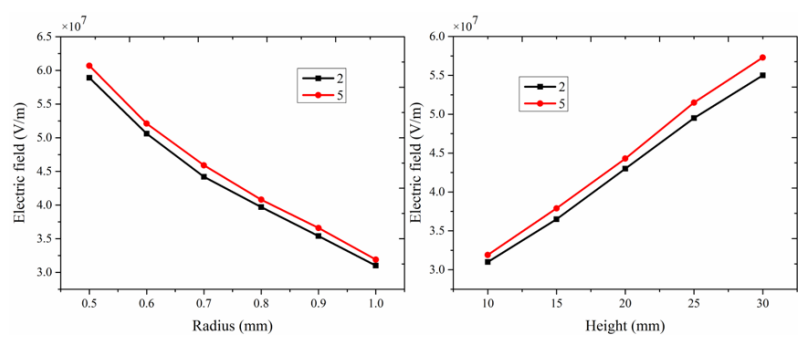

Fig. 11. Comparative study of electric field at position 2 and 5 .
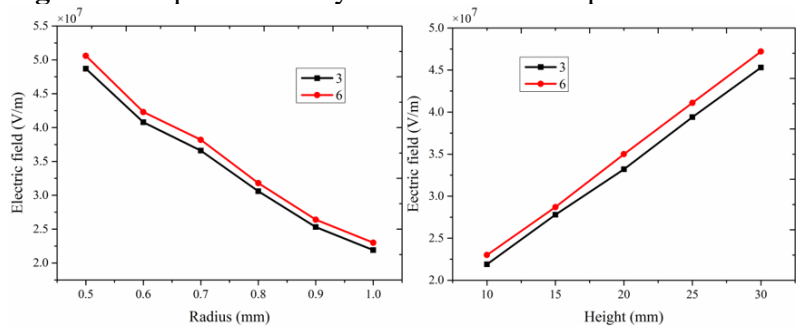

Fig. 12. Comparative study of electric field at position 3 and 6 .

We can find through the above picture that the metal protrusion on the concave side of basin-type insulator cause more serious electric field distortion than that on the convex side. Moreover, the difference is not so obvious when the metal protrusion near the high voltage conductor. However, the difference in electric field distortion on two sides increase as the metal protrusion getting closer to the grounded enclosure.

\section{Conclusions}

The 3-D simulation model of GIS with basin-type insulator was utilized to study the effect of metal protrusion on the electric field distortion in GIS. Through the calculation and analysis, we found that the metal protrusion in different positions can cause different distortion of electric field intensity. The metal protrusion near high voltage conductor lead to more severe electric field distortion than that near grounded enclosure. In addition, the electric field increases with the height of metal protrusion when the radius is constant, while decrease with the increase of radius of metal protrusion when the height is constant for all metal protrusions.

\section{References}

1. F. Yu, J. Guo, Z. Liu, D. D. Zhao, Power System Technology S2, 233 (2008)

2. X. M. Bian, S. W. Wan, Y. J. Wang, L. M. Wang, IEEJ T. Electr. Electr. 11, 133 (2016)

3. A. Q. Ma, X. Yang, X. M. Lu, X. C. Jiang, High Voltage Engineering 5, 1217 (2010)

4. K. Dreisbusch, H. G. Kranz, A. Schnettler, IEEE Trans. Dielectr. Electr. Insul. 15, 1707 (2008)

5. G. X. Zhang, B. Wang, Q. Wang, J. Z. Li, J. B. Fan, H. Tang, High Voltage Engineering 36, 335 (2010)

6. P. H. F. Morshuis, IEEE Trans. Dielectr. Electr. Insul. 12, 905 (2005) 
7. B. Zhang, Z. Qi, G. Zhang, IEEE Trans. Dielectr. Electr. Insul. 24, 1229 (2017)

8. S. Zhang, M. M. Morcos, K. D. Srivastava, IEEE Trans. Dielectr. Electr. Insul. 24, 2746 (2017)

9. D. Y. Li, W. Q. Xing, W. D. Wang, Y. G. Zhou, X. Y. Tan, Electric switchgear 4, 54 (2015) 